\title{
Differentiation therapy for IDH1/2 mutant malignancies
}

\author{
Cell Research (2013) 23:975-977. doi:10.1038/cr.2013.73; published online 4 June 2013
}

\begin{abstract}
Recently discovered recurrent somatic mutations in the key metabolic enzymes IDH1 and IDH2 produce the aberrant oncometabolite 2 -HG and contribute to malignant transformation of hematopoietic and glial cells. Two recent reports in Science describe the first IDH1 and IDH2 mutant-specific small-molecule inhibitors, which induce cell differentiation of myeloid leukemias and malignant gliomas.
\end{abstract}

Somatic mutations in epigenetic modifiers and dysregulation of the epigenetic landscape are fundamental contributors to malignant transformation. Acute Myeloid Leukemia (AML) is the most common acute leukemia affecting adults. With the notable exception of a subset of patients with Acute Promyelocytic Leukemia (APL), there has been little improvement in treatments for AML patients over the past two decades. Most patients receive cytotoxic chemotherapy and when appropriate, hematopoietic stem cell transplantation. Malignant gliomas are the most common primary brain tumors. Treatment approaches for high-grade gliomas are multifaceted and include surgery, radiation and/or chemotherapy and anti-angiogenic treatment. The majority of patients with AML and high-grade gliomas will die from their disease, thus, the development of novel therapies for both malignancies is essential.

Despite the different cellular origins of leukemias and gliomas, recent tumor sequencing efforts have identified certain recurrent somatic mutations in common between these diseases. Specifically, both diseases manifest heterozygous somatic mutations af- fecting the $\mathrm{NADP}^{+}$-dependent isocitrate dehydrogenase (IDH) enzymes [1]. IDH1 is localized to cytoplasm and peroxisomes whereas IDH2 is located in mitochondria. Both enzymes contribute to cellular pools of NADPH that is used as reductive potential in a variety of processes. IDH1 somatic mutations were first identified in malignant gliomas, affecting the R132 residue. Sequencing the first AML genome revealed similar IDH1R132 mutations. These substitutions lead to impairment in the enzyme's forward reaction and to a gain of neomorphic activity that produces the (R)-enantiomer of 2-hydroxyglutarate (2-HG) from $\alpha$-ketoglutarate $(\alpha-K G)$. IDH2 mutations at residue R172 result in similar functional outcomes. R172IDH2 enzymes have also been discovered in malignant gliomas and AML. Subsequent studies have identified additional mutations at codons encoding residues in or near the enzymes' active site. In both malignant disorders, the mutations are predictive for clinical outcome [1]. A critical role for IDH1/2 mutations and 2-HG in oncogenesis is supported by the fact that IDH $1 / 2$ mutations are common in AML and malignant gliomas, as well as other tumor types, and IDH1/2 mutations may occur early in the development of both AML and malignant gliomas.

Due to the chemical similarity between $2-\mathrm{HG}$ and $\alpha-\mathrm{KG}, 2-\mathrm{HG}$ can competitively inhibit $\alpha$-KG-dependent dioxygenases, including JmjC domaincontaining histone demethylases and the ten-eleven translocation (TET) family of DNA hydroxylases. TET loss-offunction mutations also occur in myeloid disorders. Malignant IDH1/2 and
TET mutations are generally mutually exclusive in AML, and are associated with global DNA hypermethylation and impaired hematopoietic cell differentiation, suggesting that these mutations affect the same basic molecular mechanism. IDH1/2 mutant enzymes also impair adipocyte and astrocyte differentiation [1-4]. Mutant IDH1/2 or loss-of-function TET2 induce aberrant loss of 5-hydroxymethylcytosine $(5 \mathrm{hmc})$ and increased levels of 5-methylcytosine $(5 \mathrm{mc})$. Mutant IDH1/2 may also (i) directly alter the abundance of various histone methyl marks; (ii) alter $\mathrm{NADPH} / \mathrm{NADP}^{+}$ratios, potentially leading to mitochondrial dysregulation and abnormal ROS levels; (iii) inhibit $\alpha$-KG-dependent prolyl-4-hydroxylase, leading to extracellular matrix disruption associated with the potential of tumor progression; and (iv) stabilize HIF $1 \alpha$ and hence induce transcription of genes involved in angiogenesis, cell survival, glucose metabolism and invasion $[5,6]$.

Targeting IDH1/2 mutant enzymes could thus reverse a broad range of effects caused by $2-\mathrm{HG}$ production. This could potentially result in more effective treatments for IDH1/2 mutant malignancies. Along these lines, Wang et al. [7] and Rohle et al. [8] reported small molecules that specifically inhibit two of the most common mutant IDH isotypes. High-throughput screening for mutant-specific inhibitors yielded the identificaiton of AGI-5198, which specifically inhibits R132H IDH1 mutants (R132H-IDH1), and AGI-6780, a modified urea sulfonamide, which targets the R140Q mutant IDH2 isoform (R140Q-IDH2). The authors used these 
compounds in model systems to evaluate their potential utility in the treatment of IDH1/2 mutant disorders.

Rohle et al. [8] examined AGI-5198 treatment effects in R132H-IDH1 glioma and sarcoma cells. Exposure to AGI-5198 led to a dose-dependent reduction in 2-HG production. Decreased 2-HG level was associated with tumor growth inhibition in vitro and in vivo. The requirement of R132H-IDH1 for tumor growth was confirmed in shRNA knockdown experiments. Based on evaluation of Ki67 expression and caspase- 3 cleavage, the inhibitory effect on tumor growth appeared to be due to impaired cell proliferation, rather than changes in the rate of apoptosis. AGI-5198 induced the expression of genes and cell markers associated with glial-specific differentiation in glioma cells. Consistent with previous reports suggesting a role for IDH1/2 mutants in epigenetic reprogramming $[2,3,9]$, AGI-5198 treatment in vitro resulted in reduction of repressive chromatin marks (H2K9 and H3K27 trimethylation) at promoters of induced genes. In xenograft models, $450 \mathrm{mg} / \mathrm{kg}$ AGI-5198 induced the expression of differentiation-associated genes, reduced $\mathrm{H} 3 \mathrm{~K} 9$ trimethylaytion, and caused tumor growth inhibition. However, treatment with $150 \mathrm{mg} / \mathrm{kg}$ AGI-5198 only resulted in tumor growth inhibition. This result suggests that R132H-IDH1 may have different effector mechanisms targeting cell proliferation and differentiation.

Wang et al. [7] generated a highresolution crystal structure of AGI6780 complexed with homodimeric mutant R140Q-IDH2, NADP ${ }^{+}$and $\mathrm{Ca}^{2+}$. Coupled with kinetic data, the crystalized structure suggests that AGI-6780 is a slow tight binder and functions as an allosteric inhibitor. The authors tested

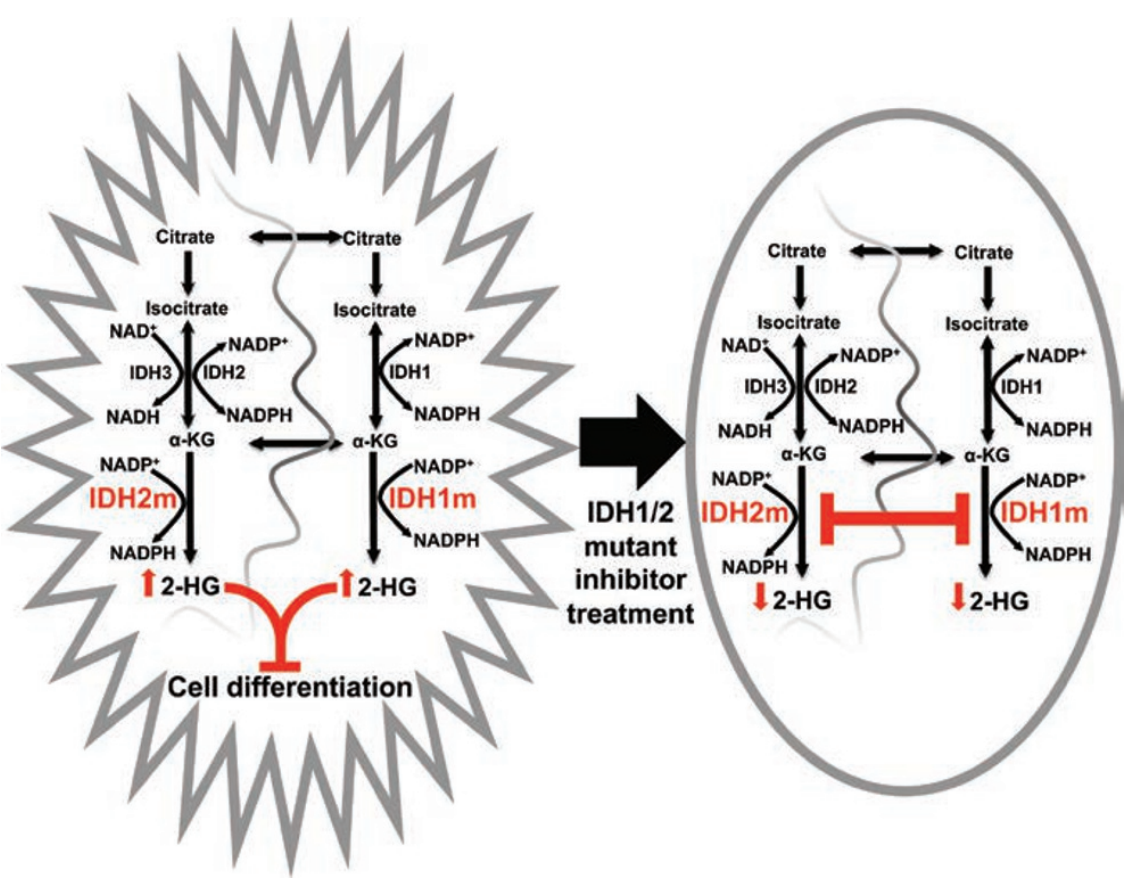

Figure $1 \mathrm{IDH} 1 / 2$ mutant enzyme inhibitors allow for restoration of cell differentiation. In IDH mutant AML and glioma cells, IDH1 (cytosol and perixosome) and IDH2 (mitochondrial) neomorphic enzymes produce the oncometabolite 2-HG. 2-HG blocks normal cell differentiation. Treatment with IDH1/2 enzyme inhibitors results in decreased 2-HG production and features consistent with cell differentiation. IDH, isocitrate dehydrogenase; IDHm, mutant IDH enzymes; 2-HG, (R)-enantiomer of 2-hydroxyglutarate; $\alpha$-KG, $\alpha$-ketoglutarate.

AGI-6780 in an R140Q-IDH2 transduced erythroleukemia cell line and ex vivo cultured R140Q-IDH2 human patient AML specimens to interrogate treatment effects. Treatment with AGI6780 reduced 2-HG levels both in cell lines and primary AML cells. AGI-6780 induced a burst in R140Q-IDH2 mutant AML cell proliferation. This was followed by a reversal in differentiation block induced by R140Q-IDH2 in the cell line and in AML cell differentiation of R140Q-IDH2 AML patient samples.

The detailed mechanisms by which AGI-5198 and AGI-6780 inhibitors affect cell cycle regulation and differentiation remain unclear. While reduction in 2-HG production likely results in reversal of metabolic aberrancies, transcriptional pattern changes leading to cell differentiation may be due to epigenetic reprogramming. Epigenetic mechanisms were suggested from histone methylation pattern changes after AGI-5198 treatment [8]. A reduction in 2-HG level could enable TET reactivation and restore normal transcriptional regulation through $5 \mathrm{hmc}$ and/or $5 \mathrm{mc}$. Furthermore, tumor growth suppression without significant changes in gene expression patterns after AGI-5198 treatment suggests the possibility of transcription-independent mechanisms [8]. More in-depth analysis of glioma and AML xenograft models are still needed to understand the critical mechanisms downstream of IDH1/2 mutations that must be reversed for anti-tumor effects to manifest. However, the data presented in these two reports show great promise for efficacy in treating IDH1/2 mutant diseases in humans.

The induction of IDH1/2 mutant tumor cell differentiation by IDH $1 / 2$ inhibitors is reminiscent of current treatment in APL (Figure 1). APL is characterized by translocations involving the retinoic acid receptor $\alpha(\mathrm{RAR} \alpha)$ gene and a differentiation block at the promyelocytic stage. All-trans retinoic acid (ATRA) disrupts the interaction of RAR $\alpha$ fusion proteins with corepressors 
and/or facilitates their degradation. ATRA can also facilitate APL blast differentiation. ATRA-based treatment regimens changed the standard care for APL from solely cytotoxic chemotherapy to differentiation therapy [10]. Today, APL is the most curable form of AML.

Malignant IDH mutations were first reported in 2008 and the development of specific inhibitors has occurred over a remarkably short period of time. Clinical testing of IDH mutant-targeted therapies in AML and gliomas is strongly justified. If malignant cell differentiation is recapitulated in humans, IDH1/2 mutant AML and glioma patients may benefit from significant improvements in clinical outcomes.

\section{Francine E Garrett-Bakelman ${ }^{1}$, Ari M Melnick ${ }^{1,2}$}

${ }^{1}$ Department of Medicine, Division of Hematology/Oncology, '2Department of Pharmacology, Weill Cornell Medical College, 1300 York Ave, New York, NY 10065, USA

Correspondence: Ari M Melnick

E-mail: amm2014@med.cornell.edu

\section{References}

1 Dang L, Jin S, Su SM. Trends Mol Med 2010; 16:387-397.
2 Figueroa ME, Abdel-Wahab O, Lu C, et al. Cancer Cell 2010; 18:553-567.

3 Lu C, Ward PS, Kapoor GS, et al. Nature 2012; 483:474-478.

4 Losman JA, Looper RE, Koivunen P, et al. Science 2013; 339:1621-1625.

5 Shih AH, Abdel-Wahab O, Patel JP, et al. Nat Rev Cancer 2012; 12:599-612.

6 Sasaki M, Knobbe CB, Itsumi M, et al. Genes Dev 2012; 26:2038-2049.

7 Wang F, Travins J, Delabarre B, et al. Science 2013; 340:622-626.

8 Rohle D, Popovici-Muller J, Palaskas N, et al. Science 2013; 340:626-630.

9 Sasaki M, Knobbe CB, Munger JC, et al. Nature 2012; 488:656-659.

10 Wang ZY, Chen Z. Blood 2008; 111:25052515. 